\title{
Lifetime modelling of electrical machines using the methodology of design of experiments
}

\author{
Lucas Vincent Hanisch ${ }^{1 *}$, Markus Henke ${ }^{1}$ \\ ${ }^{1}$ Inst. for Electrical Machines, Tractions and Drives, Technische Universität Braunschweig, Hans-Sommer Str. 66, \\ 38118 Braunschweig, Germany; */-v.hanisch@tu-braunschweig.de
}

\begin{abstract}
In the coming years, electromobility will be confronted with increasing demands regarding the reliability of electrical machines. In this paper a modeling methodology is presented, which allows to estimate the reliability and lifetime of the insulation system of electrical machines. Different statistical and physical modeling methods are presented, which are transformed for the later multiple regression. The methodology of Design of Experiments (DoE) is used to describe the insulation system. Since the effort for the experimental design of the DoE varies strongly with the number of effects to be investigated and the statistical accuracy, different experimental designs are presented, which can be considered for different numbers of factors. Depending on the research question, a suitable experimental design can be selected. For the calculation of the lifetime, Miner's rule is used in addition to the multiple regression, so that the percentage lifetime consumption due to a load spectrum can also be calculated.
\end{abstract}

\section{Introduction}

In the future, technologies such as electrified aircraft, trolley wire trucks or autonomous driving will be established in the field of electric mobility. Reliability, durability and safety are important criteria for the acceptance of new technologies in society. In addition to increased safety requirements, these technologies must also be able to cope with new, more challenging boundary conditions.

Whereas bearing damage used to be the most frequent cause of failure of electrical machines, the increased requirements and new boundary conditions lead to a more varied error pattern [1], [2]. A deep understanding of the causes of failure and the relevant damage mechanisms is necessary to design electrical machines for these new applications. In addition to the design of durable machines that operate under increased environmental conditions, lifetime models, on the other hand, can help electrical machines achieve a minimum target lifetime for a given load. This offers potential to save resources and reduce costs. Lifetime models and reliability analyses are therefore becoming increasingly important in the design process of electrical machines.

\section{Damage mechanisms}

The damage of electrical machines is caused by various mechanisms from different physical disciplines and there are various modelling approaches to model these damage processes. Since the use of wide bandgap semiconductors and the increasing electrical load, the cause of failure of electrical machines is increasingly based on faults and breakdowns of the electrical insulation system [2]. Because failures of the electrical insulation system will occure more frequently in the future, research concentrates on lifetime models of these insulation systems.

Basically, with regard to the aging effects that damage the insulation system, a distinction can be made between constant stresses and transient stresses. The probability of a fault in the insulation system at constant stresses is proportional to the number of operating hours and at transient stresses proportional to the number of transient effects. Figure 1 shows as examples for constant stresses the ambient temperature and the voltage slope oval and for transient stresses partial discharges rectangular.

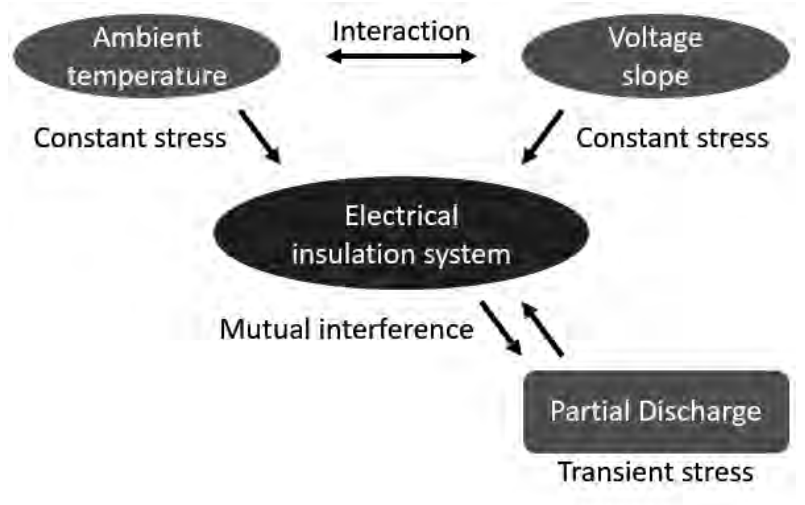

Figure 1: Examples of different stresses and their interactions.

A breakdown in the insulation system often occurs due to different combinations of the individual stresses or the interactions of these stresses. 
As an example for the interaction of stresses figure 1 shows the interaction between the temperature and the voltage slope. Insulating materials heat up due to dielectric losses. The dielectric power loss as well as the dielectric loss factor are again frequency dependent. Power electronic signals with high voltage slope and high frequency harmonics can therefore influence the temperature of the insulation system.

An interaction can also become effective by changing the properties of the insulation system. Partial discharges can damage the insulation system and lead to air-filled cavities. Due to the low permittivity of air and the significantly lower dielectric strength of air compared to insulation materials, increased field strengths in these cavities can intensify partial discharges and the aging of the insulation system. In [3] an interaction due to changed properties of the insulation system is called indirect interaction.

\section{Modeling methodologies}

The modelling approaches can be divided into two different categories. The physical and the statistical approaches.

\subsection{Physical modeling approaches}

The goal of physical modelling approaches is the mathematical description of the aging effects. A holistic model, in which the different aging mechanisms from different physical disciplines are integrated, is very complex. In addition, at certain activation energies additional phenomena occur, become more dominant or the damage effect has to be described mathematically completely different.

The advantage compared to statistical models is that no lifetime tests are necessary for the parameterization of the model. Only the measurement of single physical quantities is necessary.

One of the first physical models to describe aging effects was developed by Crine and was originally used for extrudet dielectric cables. Crine takes up Artbauer's theory and assumes that the dielectric strength of amorphous insulation materials is essentially determined by the presence of vacancies induced by electromechanical deformation of molecular chains in the insulation material. In these vacancies, the free electrons find more favourable conditions to absorb the energy necessary for impact ionization due to the applied electric field [4]. Crine assumes that above a critical field strength, the number of these vacancies increases and they combine to form larger submicrocavities. When submicrocavities have formed, electrons or ions are strongly accelerated under the influence of the electric field and can absorb enough kinetic energy to break weak Van der Waals bonds. As the submicrocavities expand, the electrons absorb even more energy and break more molecular bonds until the insulating material finally collapses and electrical breakdown occurs [5].

Crine describes the probability of breaking Van der Waals bonds depending on temperature $T$ and electric field strength $E$ as follows:

$$
p^{+}(T, E) \cong \frac{k T}{h} \exp \left(-\frac{\Delta G-e \lambda E}{k T}\right)
$$

Here $k$ is the Boltzmann constant, $h$ the Planck's constant, $\Delta G$ the critical field strength, $e$ the charge of an electron and $\delta$ the length of the free path. Additionally Crine considers the probability of the backward process as

$$
p^{-}(T, E) \cong \frac{k T}{h} \exp \left(-\frac{\Delta G+e \lambda E}{k T}\right)
$$

The net destruction rate is the subtraction of these probabilities

$$
p=p^{+}-p^{-} \cong \frac{2 k T}{h} \exp \left(-\frac{\Delta G}{k T}\right) \sinh \left(\frac{e \delta E}{k T}\right)
$$

In Crines physical model, the lifetime of the insulation system is given as the reciprocal of the destruction rate

$$
L \cong \frac{h}{2 k T} \exp \left(\frac{\Delta G}{k T}\right) \operatorname{csch}\left(\frac{e \lambda E}{k T}\right)
$$

At high fields, equation (4) reduces to

$$
L \cong \frac{h}{2 k T} \exp \left(\frac{\Delta G-e \lambda E}{k T}\right)
$$

After Crine, Lewis developed a new physical model that is also based on the formation of microcavities and the breaking of molecular bonds. Just like Crine, Lewis also takes into account the temperature $T$ and the electric field strength $E$. The destruction rate $K_{\mathrm{b}}$ and the formation rate $K_{\mathrm{r}}$ of the bonds are determined as follows:

$$
\begin{aligned}
& K_{b}(T, E)=\frac{k T}{h} \exp \left(-\frac{U_{b}-\gamma_{b} \varepsilon E^{2}}{k T}\right) \\
& K_{r}(T, E)=\frac{k T}{h} \exp \left(-\frac{U_{r}+\gamma_{r} \varepsilon E^{2}}{k T}\right)
\end{aligned}
$$

$U_{\mathrm{b}}$ and $U_{\mathrm{r}}$ are the critical energies at which the bonds break or form again. $\varepsilon$ is the dielectric permittivity and $\gamma_{\mathrm{b}}$ or $\gamma_{\mathrm{r}}$ are fitting parameters with the dimensions of a volume. The aging of the insulation system is equated with the propagantion of cracks and voids in the insulation system according to the Griffith criterion [6] 


$$
L=\int_{0}^{b_{c}} \frac{1}{K_{b}(1-b)-K_{r} b} d b
$$

$b_{c}$ represents the critical number of broken molecular bonds above which insulation failure occurs.

\subsection{Statistical modeling approaches}

In contrast to physical models, statistical lifetime models are based on accelerated lifetime tests. The most popular statistical lifetime model was developed by Arrhenius and describes the quantitative dependence of the chemical reaction rate $r$ on the temperature $T$

$$
r=A \cdot e^{-\frac{E_{A}}{k T}}
$$

where $A$ is a constant and $E_{\mathrm{A}}$ is the activation Energy. Dakin used Arrhenius' equation to describe the processes taking place in the insulation material as a function of temperature. Except for the constants Dakin uses the same equation as Arrhenius [7]:

$$
L=A_{\text {Dakin }} \cdot e^{\frac{B_{\text {Dakin }}}{T}}
$$

Partial discharges are often cited in the literature as the cause of electrical aging of electrical insulation systems. Their frequency and effects on service life increase exponentially with increasing voltage $V$. Mathematically, this realationship can be described using the inverse power model

$$
L=c \cdot V^{-n}
$$

here $c>0$ is a material constant and $n>0$ is the power law constant.

\section{Design of Experiments}

As mentioned before, the parameterization of the models from chapter 2.2 is done by accelerated lifetime tests. Despite the increased conditions compared to real operation, these tests are complex and time consuming. Physical models, on the other hand, are mathematically very complex and often only consider the electric field strength and temperature. The physical modelling of additional aging effects and the modelling of the individual interactions leads to an exponential increase in complexity and to the failure of the purely physical modelling approach. With the methodology of Design of Experiments (DoE) it is possible to model many effects including their interactions and to reduce the number of necessary life tests to a minimum.

The basic idea is that the insulation system, or even any deterministic system, can be described with a mathematical model. Different approaches can be used as mathematical models. Some were presented in chapter 2. The parameterization of the model is done after the complete evaluation of the experimental design. DoE is therefore one of the statistical model approaches. In the following different experimental designs are discussed.

\subsection{Full factorial design}

In an experimental design, the number of effects to be investigated, the number of measuring points and the number oof measured values per measuring point are defined. The advantage of an experimental design compared to a one-factor-at-a-time-plan is that each test result can be used to calculate several effects. This considerably reduces the test effort.

If, for example, eight measured values per measureing point are necessary to determine the influence of a factor on the service life of the insulation system with acceptable accuracy, a "one factor at a time" plan requires 32 tests. In the first 8 tests the lifetime would be determined for the factors $x_{1}, x_{2}$ and $x_{3}$ at a low level. Then eight tests would be carried out on each factor at an increased level to investigate the influence of a factor on the service life. This is shown in figure 2a. With a full factorial design, 16 tests would be sufficient to determine the effect of a factor with the same accuracy. Instead of examining the effect once by comparing eight value pairs, the effect is examined four times by comparing two value pairs. The number of value pairs used to calculate an effect and the statistical validation are identical. Figure $2 \mathrm{~b}$ shows the full factorial design. Since the test specimens can only be used once in the accelerated lifetime tests, the full factorial design reduces costs and time. In addition, the information content obtained from the measurements is increased, because the effect of the factors can be analyzed at different levels.
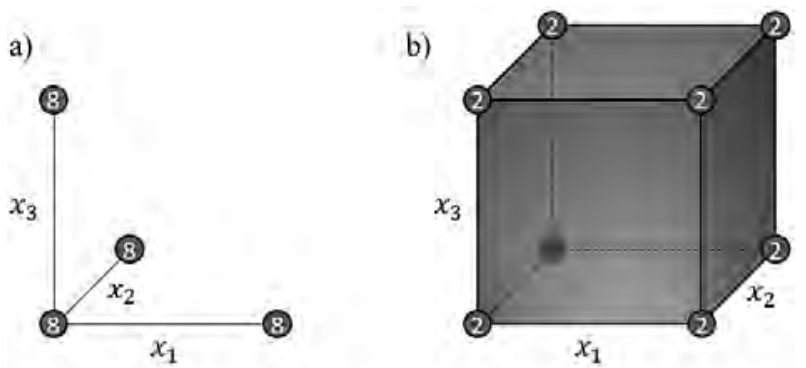

Figure 2: Comparison of a "one factor at a time" plan a) with a full factorial design $b$ ).

With each additional factor added to an experimental 
design, the information content of the DoE increases. With increasing information content, the effort of the experimental design increases with $F^{\mathrm{m}}$. $F$ is the number of measuring points in one dimension and $m$ the number of factors. The experimental design in figure $2 \mathrm{~b}$ has $2^{3}=8$ measuring points. The number of measurements per measuring point determines the accuracy of the experimental design. The experimental design in figure $2 b$ has a total of 16 measurement values with which the influence of the effects on the service life of the insulation system can be determined. If the number of tests per measurement point is increased from two to eight, 64 measurement values can be used. The accuracy increases, but also the effort. Information content, accuracy and effort of an experimental design are inseparably linked. The optimal experimental design must be adapted to the respective requirements. Figure 3 shows the combination of the three basic properties of an experimental design. If the point is further outside the triangle, an increase in information, an increase in accuracy or a reduction in effort is meant.

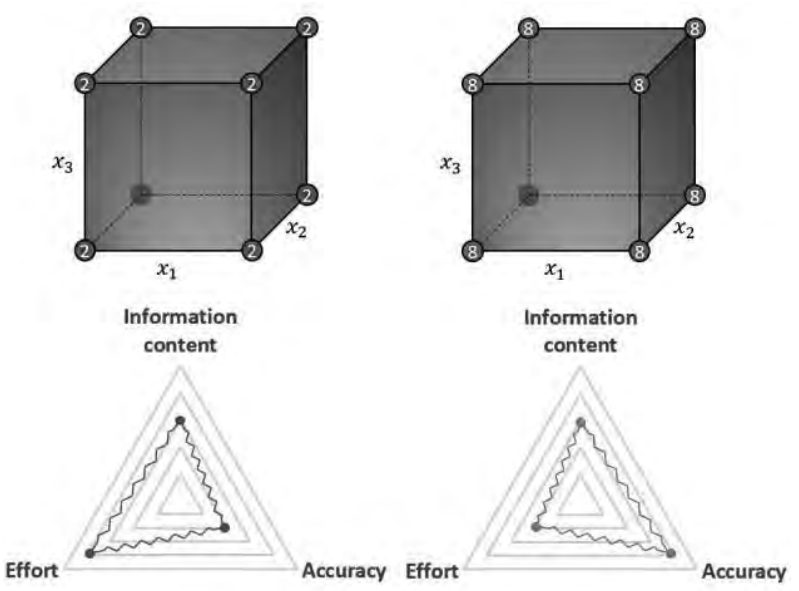

Figure 3: Variation of an experimental design by an increased number of tests.

With statistical models there is always the risk of errors. There are two possible types of errors that can occur. The influence of a factor on the aging of the insulation system could be assumed to be significant, although in reality it is not responsible for the aging or a significant effect on the aging of an insulation system could be overlooked. In a hypothesis test these errors are called first type errors and second type errors. In order to determine the number of test specimens required in a design for a given accuracy, such a hypothesis test should be performed.

The hypothesis is as follows: The factor has no effect on the lifetime of the insulation system. In table 1 the four possible decisions of this hypothesis test are shown. The accuracy of the experimental design is determined by the errors of the first $\alpha$ and second type $B$ and the change in lifetime $\Delta L$ to be detected. Table 2 shows the number of tests to achieve the desired accuracy.

\begin{tabular}{c|c|c|}
\cline { 2 - 3 } \multicolumn{1}{c|}{$\begin{array}{c}\text { Assume } \\
\text { hypothesis }\end{array}$} & $\begin{array}{c}\text { Non-significant effect } \\
\text { detected }\end{array}$ & $\begin{array}{c}\text { Hypothesis wrong } \\
\text { Type I error } \boldsymbol{\alpha} \\
\text { non-significant effect } \\
\text { assumed to be significant }\end{array}$ \\
\cline { 2 - 3 } $\begin{array}{c}\text { Reject } \\
\text { hypothesis }\end{array}$ & $\begin{array}{c}\text { Type II error } \boldsymbol{\beta} \\
\text { Significant effect not } \\
\text { recognized }\end{array}$ & $\begin{array}{c}\text { Power }(1-\boldsymbol{\beta}) \\
\text { Significant effect detected }\end{array}$ \\
\hline
\end{tabular}

Table 1: Possibilities of a hypothesis test.

\begin{tabular}{|c|c|c|c|c|c|c|c|c|c|c|c|c|c|c|}
\hline & \multicolumn{4}{|c|}{$\alpha=10 \%$} & \multicolumn{4}{|c|}{$\alpha=5 \%$} & \multicolumn{5}{|c|}{$\alpha=1 \%$} & \multirow{2}{*}{\begin{tabular}{|c|} 
Type I error \\
Power \\
\end{tabular}} \\
\hline$\Delta \mathrm{L} / \sigma$ & $60 \%$ & $70 \%$ & $80 \%$ & $90 \%$ & $60 \%$ & $70 \%$ & $80 \%$ & $90^{\circ}$ & 60 & & \begin{tabular}{l|l}
$\%$ & 8
\end{tabular} & $80 \%$ & \begin{tabular}{|l|}
$90 \%$ \\
\end{tabular} & \\
\hline 0,5 & 60 & 78 & 102 & 140 & 82 & 102 & 128 & 17 & 13 & & 58 & 192 & 242 & \\
\hline 0,75 & 28 & 36 & 46 & 64 & 38 & 46 & 58 & 78 & 62 & & 2 & 88 & 110 & \\
\hline 1 & 16 & 22 & 28 & 36 & 22 & 28 & 34 & 46 & 36 & & 2 & 52 & 64 & \\
\hline 1,5 & 10 & 12 & 14 & 18 & 12 & 14 & 18 & 22 & 18 & & 2 & 26 & 30 & \\
\hline 2 & 6 & 8 & 8 & 12 & 8 & 10 & 12 & 14 & 12 & & 4 & 16 & 20 & \\
\hline
\end{tabular}

Table 2: Necessary number of tests for a certain accuracy when each factor has two levels.

If a significant effect is to be detected at $(1-\beta)=$ $90 \%$, if it changes the lifetime by at least one standard deviation $\sigma$ on average, and a non-significant effect is to be falsely assumed to be significant only at $\alpha=1 \%, 64$ tests are required for this experimental design. This corresponds to the experimental design shown in figure 3 on the right. The experimental design in figure 3 left is less complex with 16 tests, but a significant effect is detected only at $(1-\beta)=60 \%$ and the probability of erroneously assuming a non-significant effect as significant is $\alpha=10 \%$. Since the standard deviation $\sigma$ influences the accuracy of the DoE, the test specimens should be manufactured as identically as possible and the accelerated lifetime tests should be performed under the same boundary conditions.

\subsection{Fractional factorial design}

Not only a high accuracy requires a high number of tests but also with increasing information content and the consideration of further influencing factors the effort of an experimental design increases with $F^{\mathrm{m}}$. One possibility to efficiently investigate several effects despite the exponentially growing effort is the use of fractional factorial designs. The correlation between information content, accuracy and effort from figure 3 can not be avoided, but 
in experimental designs that take many factors into account, some information is irrelevant or can be excluded in advance as a cause for the aging of the insulation system. This is especially true for the $i$-fold interactions between the factors $m$, which can be calculated as follows

$$
\left(\begin{array}{c}
m \\
i
\end{array}\right)=\frac{m !}{i ! \cdot(m-i) !}
$$

The larger $m$ the more interactions are predominantly investigated.

To investigate the effect of a factor on the lifetime of the insulation system is the primary goal of the lifetime model. Also the effect of the interaction of two factors on the lifetime is of interest. The influence of higher interactions is physically difficult to assess and often negligible compared to two-fold interactionsor simple effects. Instead of these higher interactions additional single or double interactions could be investigated. In the case of fractional factorial designs not all measureing points are executed. Figure 4 shows the comparison of full factorial design and a fractional factorial design.

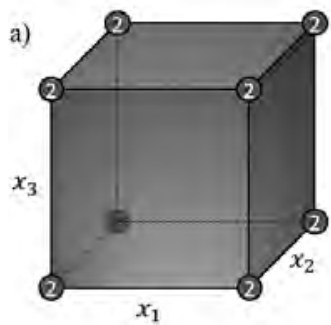

b)

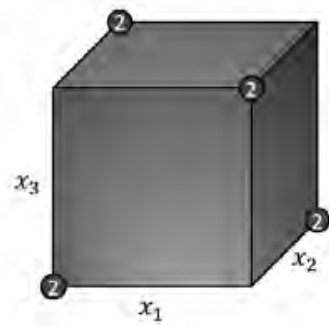

Figure 4: Comparison of a full factorial design a) and a fractional factorial design $b$ ).

This leads to a loss of information and to the fact that individual effects mix with each other. It is later not possible to distinguish which of these mixed effects is responsible for the aging of the insulation system. However, if simple factors and two-fold interactions are mixed exclusively with higher interactions whose effect on the lifetime is negligible, the question of how to distinguish between these effects is unnecessary. Table 3 lists and evaluates various fractional factorial designs.

\section{Lifetime modeling}

After different modelling methods were described in chapter 2 and the DoE was described in chapter 3, this chapter focuses on the development of the lifetime model based on the DoE.

\subsection{Multiple regression}

As a mathematical model to describe the insulation system, multiple regression is used. With this model it is possible to adapt the relationship between the influencing variables $x_{\mathrm{i}}$ and the lifetime as target variable $y$ to the measured values. For the description a full factorial design with two measured values per measuring point is used, as shown in figure 4a. The formula for the calculation of the lifetime is given with the multiple regression as follows

$$
\begin{aligned}
y= & z_{0}+z_{1} x_{1}+z_{2} x_{2}+z_{3} x_{3}+z_{12} x_{12}+ \\
& z_{13} x_{13}+z_{23} x_{23}+z_{123} x_{123}
\end{aligned}
$$

where $z_{\mathrm{i}}$ is the influence of factor $x_{\mathrm{i}}$. The measured values required to parameterize equation (13) are designated $c_{\mathrm{i}}$ in table 4. In the experimental design in figure 4a, each factor has two levels, designated by the values -1 for the lower level and 1 for the higher level in table 4. For two measured values, the mean value could also be used instead of the expected value. If there are several measured values, the expected value should be used. The measured

\begin{tabular}{|c|c|c|c|c|c|c|c|c|c|c|c|}
\hline \begin{tabular}{|c|}
$\begin{array}{c}\text { Measuring } \\
\text { points }\end{array}$ \\
\end{tabular} & Constant & & & & $x_{12}$ & $x_{13}$ & $x_{23}$ & $x_{123}$ & \begin{tabular}{|c|} 
Measured \\
value 1 \\
\end{tabular} & $\begin{array}{c}\text { Measured } \\
\text { value 2 } \\
\end{array}$ & $\begin{array}{c}\text { Expected } \\
\text { value } \\
\end{array}$ \\
\hline 1 & 1 & -1 & -1 & -1 & 1 & 1 & 1 & -1 & $c_{11}$ & $c_{12}$ & $y_{1}$ \\
\hline 2 & 1 & -1 & -1 & 1 & 1 & -1 & -1 & 1 & $c_{21}$ & $c_{22}$ & $y_{2}$ \\
\hline 3 & 1 & -1 & 1 & -1 & -1 & 1 & -1 & 1 & $c_{31}$ & $c_{32}$ & $y_{3}$ \\
\hline 4 & 1 & -1 & 1 & 1 & -1 & -1 & 1 & -1 & $c_{41}$ & $c_{42}$ & $y_{4}$ \\
\hline 5 & 1 & 1 & -1 & -1 & -1 & -1 & 1 & 1 & $c_{51}$ & $c_{52}$ & $y_{5}$ \\
\hline 6 & 1 & 1 & -1 & 1 & -1 & 1 & -1 & -1 & $c_{61}$ & $c_{62}$ & $y_{6}$ \\
\hline 7 & 1 & 1 & 1 & -1 & 1 & -1 & -1 & -1 & $c_{71}$ & $c_{72}$ & $y_{7}$ \\
\hline 8 & 1 & 1 & 1 & 1 & 1 & 1 & 1 & 1 & $c_{81}$ & $c_{82}$ & $y_{8}$ \\
\hline
\end{tabular}
values in life cycle investigation are usually Weibull distributed.

Table 4: Necessary number of tests for a certain accuracy when each factor has two levels.

To determine the influence of the factors zi on the lifetime of the insulation system, equation (13) can be converted to matrix notation

$$
\underline{Z}=\underline{X}^{-1} \cdot \underline{Y}_{i}
$$

$\underline{X}$ and $\underline{Y}_{i}$ are shown in green and blue in table 4 . The relation of the individual factors with the lifetime of the insulation system is not linear but can be described with the models from chapter two. These non-linear correlations must be transformed and used in the linear equation (13). In equation (15) this is represented for three factors which were described with the statistical approaches from chapter 2.2 


\begin{tabular}{|c|c|c|c|c|c|c|c|c|c|c|c|c|c|}
\hline Measuring points & 3 & 4 & 5 & 6 & 7 & 8 & 9 & 10 & 11 & 12 & Resolution & Mix & Evaluation \\
\hline 4 & $2^{3-1}$ & & & & & & & & & & 1 & Factor and 2-fold & critical \\
\hline 8 & \begin{tabular}{|c|}
$2^{3}$ \\
complete
\end{tabular} & $\begin{array}{l}2^{4-1} \\
\text { II }\end{array}$ & $2^{5-2}$ & $\begin{array}{l}2^{6-3} \\
1\end{array}$ & $\begin{array}{l}2^{7-4} \\
1\end{array}$ & & & & & & ॥ & $\begin{array}{l}\text { Faktor and 3-fold } \\
\text { 2-fold and 2-fold }\end{array}$ & critical \\
\hline 16 & & \begin{tabular}{c|}
$2^{4}$ \\
complete
\end{tabular} & $\begin{array}{l}2^{5-1} \\
\text { III }\end{array}$ & $\begin{array}{c}2^{6-2} \\
I 1\end{array}$ & $\begin{array}{c}2^{7-3} \\
\text { II }\end{array}$ & $\begin{array}{l}2^{8-4} \\
\text { II }\end{array}$ & $\begin{array}{l}2^{9-5} \\
1\end{array}$ & $\begin{array}{l}2^{10-6} \\
1 \\
\end{array}$ & $\begin{array}{c}2^{11-7} \\
\mathrm{I}\end{array}$ & $\begin{array}{c}2^{12-8} \\
1 \\
\end{array}$ & III & $\begin{array}{l}\text { Factor and 4-fold } \\
\text { 2-fold and } 3 \text {-fold }\end{array}$ & uncritical \\
\hline 32 & & & $\begin{array}{c}2^{5} \\
\text { complete }\end{array}$ & $\begin{array}{l}2^{6-1} \\
\text { IV }\end{array}$ & $\begin{array}{c}2^{7-2} \\
\|\end{array}$ & $\begin{array}{l}2^{8-3} \\
\text { II }\end{array}$ & $\begin{array}{c}2^{9-4} \\
\text { II }\end{array}$ & $\begin{array}{c}2^{10-5} \\
\text { II }\end{array}$ & $\begin{array}{l}2^{11-6} \\
\text { II }\end{array}$ & $\begin{array}{l}2^{12-7} \\
\text { II }\end{array}$ & IV & higher resolution & uncritical \\
\hline 64 & & & & \begin{tabular}{|c|}
$2^{6}$ \\
complete \\
\end{tabular} & 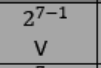 & $\begin{array}{c}2^{8-2} \\
\text { III }\end{array}$ & $\begin{array}{c}2^{9-3} \\
\text { II }\end{array}$ & $\begin{array}{c}2^{10-4} \\
\text { II }\end{array}$ & $\begin{array}{l}2^{11-5} \\
\text { II }\end{array}$ & $\begin{array}{c}2^{12-6} \\
\text { II }\end{array}$ & $\mathrm{v}$ & higher resolution & uncritical \\
\hline 128 & & & & & $\begin{array}{c}2^{7} \\
\text { complete }\end{array}$ & $\begin{array}{l}2^{8-1} \\
\text { VI }\end{array}$ & $\begin{array}{l}2^{9-2} \\
\text { IV }\end{array}$ & $\begin{array}{c}2^{10-3} \\
\text { III }\end{array}$ & $\begin{array}{c}2^{11-4} \\
\text { III }\end{array}$ & $\begin{array}{c}2^{12-5} \\
\text { II }\end{array}$ & VI & higher resolution & uncritical \\
\hline
\end{tabular}

Table 3: Overview and evaluation of fractional factorial designs for the lifetime of the insulation system [8].

$$
\begin{aligned}
\log y= & z_{0}+z_{1} \cdot e^{B_{\text {Dakin }} \cdot x_{1}}+z_{2} \cdot \log x_{2}+ \\
& z_{3} \cdot \log x_{3}+z_{12} \cdot e^{B_{\text {Dakin }} \cdot x_{1}} \cdot \log x_{2}+ \\
& z_{13} \cdot e^{B_{\text {Dakin }} \cdot x_{1}} \cdot \log x_{3}+ \\
& z_{23} \cdot \log x_{2} \cdot \log x_{3}+ \\
& z_{123} \cdot e^{B_{\text {Dakin }} \cdot x_{1}} \cdot \log x_{2} \cdot \log x_{3}
\end{aligned}
$$

The effect of the individual factors and the respective interaction, can be read off at the parameters $Z_{\mathrm{i}}$. A comparison of these parameters provides information about which effect contributes significantly to the aging of the insulation system and which effects are negligible with respect to service life. When designing robust insulation systems, special attention should be paid to the effects with a high contribution to aging.

\subsection{Miner's rule}

With the mathematical description of the insulation system by multiple regression and the efficient parameterization with the DoE, the lifetime of the insulation system at a certain load can be estimated. The lifetime model will now be extended to estimate the percentage of lifetime consumption due to a variable load spectrum. This is especially useful for applications with strong load fluctuations such as in the automotive industry.

Using the Miner's rule, the lifetime consumption of individual loads of a load spectrum can be calculated. It is assumed that the insulation system is loaded with a constant load for a short period of time. The duration of the constant load $l_{\mathrm{i}}$ is related to the total lifetime of the insulation system at the same load $L i$. The sum of the individual loads result in the percentage lifetime consumption. The closer the sum of the individual loads approaches the value 1 , the more lifetime is consumed and the more likely the insulation system is to fail. Miner's rule is given in equation (16)

$$
\sum_{i=1}^{n} \frac{l_{i}}{L_{i}}=L_{\%}
$$

where $L \%$ is the percentage lifetime consumption.

\section{Conclusion}

In this paper a methodology for the lifetime calculation of the insulation system of electrical machines is presented. Besides the prediction of the lifetime, the effects of individual factors and the interactions considered in the modelling can be evaluated and compared. The model can be used in the design process of electrical machines or can be used as a virtual test bench, where the lifetime can be investigated at different loads. Figure 5 shows an overview of the entire methodology. The WLTP is shown as an example of the load spectrum, since the model can be used particularly well in the automotive industry.

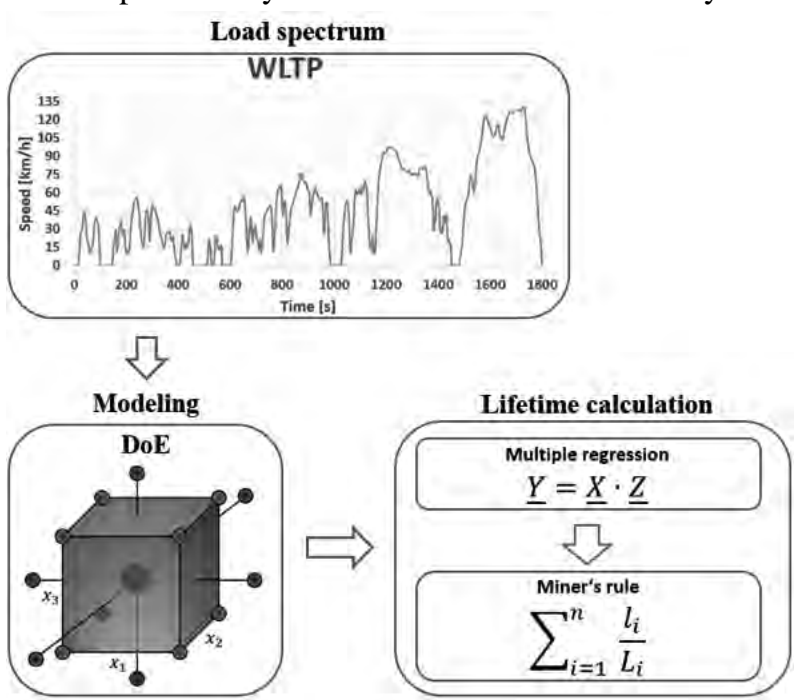

Figure 5: Overview of the modelling methodology.

Currently, durability tests are being carried out to apply the model to a real machine. Since the focus of this article 
is on modelling, the results and aging effects will be published in a separate article.

\section{References}

[1] A. H. Bonnett and C. Yung, "Increased Efficiency Versus Increased Reliability," in IEEE Industry Applications Magazine, vol. 14, no. 1, pp. 29-36, Jan.-Feb. 2008, doi: 10.1109/MIA.2007.909802.

[2] R. Brütsch, M. Tari, K. Fröhlich, T. Weiers and R. Vogelsang, "Insulation Failure Mechanisms of Power Generators [Feature Article]," in IEEE Electrical Insulation Magazine, vol. 24, no. 4, pp. 17-25, July-Aug. 2008, doi: 10.1109/MEI.2008.4581636.

[3] A. C. Gjerde, "Multifactor ageing models - origin and similarities," in IEEE Electrical Insulation Magazine, vol. 13, no. 1, pp. 6-13, Jan.-Feb. 1997, doi: 10.1109/57.567392.

[4] J. Artbauer,"Electric Strength of Polymers," Journal of Physics D, Appl. Phys., Vol. 29, pp. 446-56, 1996.

[5] J. -. Parpal, J. -. Crine and Chinh Dang, "Electrical aging of extruded dielectric cables. A physical model," in IEEE Transactions on Dielectrics and Electrical Insulation, vol. 4, no. 2, pp. 197-209, April 1997, doi: 10.1109/94.595247.

[6] G. Pietrini, D. Barater, F. Immovilli, A. Cavallini and G. Franceschini, "Multi-stress lifetime model of the winding insulation of electrical machines," 2017 IEEE Workshop on Electrical Machines Design, Control and Diagnosis (WEMDCD), Nottingham, 2017, pp. 268-274, doi: 10.1109/WEMDCD.2017.7947758.

[7] T. W. Dakin, "Electrical Insulation Deterioration Treated as a Chemical Rate Phenomenon," in Transactions of the American Institute of Electrical Engineers, vol. 67, no. 1, pp. 113-122, Jan. 1948, doi: 10.1109/TAIEE.1948.5059649.

[8] W. Kleppmann, "Versuchsplanung Produkte und Prozesse optimieren”, 2013 Carl Hanser Verlag München Wien, ISBN, 978-3-446-43752-4. 